US Army Corps of Engineers $\mathbb{\circledast}_{\circledast}$

\section{Modeling Free-Surface Flow Over a Weir}

by Richard Stockstill, Christopher Kees, and Charlie Berger

PURPOSE: This Coastal and Hydraulics Engineering Technical Note (CHETN) investigates the ability of a modeling scheme to compute not only the flow variables (pressure and velocity), but also the location of the free surface. The free-surface scheme is implemented in the threedimensional (3-D) Navier-Stokes module of the Adaptive Hydraulics (ADH) code and provides a means to model free-surface flow over a hydraulic structure. A review of existing methods of freesurface tracking is provided. A particular form of a moving-mesh algorithm is tested by comparing simulation results of flow over a weir with published data.

BACKGROUND: In modeling the hydrodynamics of water bodies, the water surface can be regarded as a free surface or as a rigid lid. Treatment of the free surface is discussed in the following paragraphs. Treating the surface as a rigid lid (solid boundary) requires that the pressure be computed at this boundary. Modeling the water surface as a rigid plane is reasonable for large, deep bodies of water where the Froude number is small; but it is usually not appropriate for flow over hydraulic structures where significant variations in the water surface are expected.

Calculation of the free surface in 3-D Navier-Stokes models may be complicated, depending on the hydraulic conditions of the problem at hand. Aliabadi et al. (2003) review methods used to address the free-surface issue in multidimensional modeling. They report two methods for representing a moving free surface: the fixed-mesh technique and the moving-mesh technique. The choice of technique depends on the complexity of the expected free-surface shape. The question is whether small water-surface displacement is expected, or whether breaking waves and hydraulic jumps are expected. Moving-mesh algorithms employ a kinematic boundary condition on the free surface such that the mesh moves with the free surface. A commonly used technique is the Arbitrary Lagrangian-Eulerian (ALE) description (Donea 1982; Farhat et al. 1995). Another moving-mesh method uses space-time finite element formulations of the governing equations (Gulcat et al. 1997; Tezduyar et al. 1992). Behr (2001) used the space-time finite element approach to capture the contorted, time-dependent free surface of flow passing over a fixed-crest weir. Fixed mesh methods include the popular volume of fluid (VoF) (Hirt and Nichols 1981), the level set method (LSM) (Osher and Sethian 1988; Sussman et al. 1994; Sethian 1999; Chang et al. 1996; and Lin et al. 2005), and the interface-sharpening/global mass conservation (IS-GMC) methods (Aliabadi et al. 2003).

The VOF method and LSM do not determine the water surface position explicitly. The primary advantage of these fixed mesh methods is that the water surface need not be smooth or even single-valued. These methods are robust even for their contorted surface patterns including wave breaking. Moving mesh methods can be posed to provide accurate water-surface location, but at the risk of mesh tangling (or element shearing). The recent paper by Lin et al. (2005) illustrates the 
applicability of the LSM to mechanical and civil engineering applications. They show that LSM can be customized for solution of flow over hydraulic structures.

Aliabadi et al. (2003) have combined moving-mesh and fixed-mesh techniques in a finite element context to simulate the physical motion of moving objects influenced by the flow field, leading to a coupling of fluid/structure interaction. For surface evolution they use the interface-sharpening/ global mass conservation (IS-GMC) approach, which is a variant of the VoF approach designed primarily to avoid the somewhat diffuse phase interfaces that simple VoF methods tend to produce while maintaining the discrete mass conservation properties characteristic of VoF.

APPROACH: The 3-D Navier-Stokes module of the ADH flow solver is used to model the velocities, pressures, and water-surface elevations of the flow approaching and overtopping a removable spillway weir (RSW). ADH is a suite of finite element models of which, the NavierStokes module solves the Reynolds-Averaged Navier-Stokes (RANS) equations using the method of finite elements. ADH has been applied to flows approaching the Ice Harbor and Lower Monumental Dams (Stockstill et al. 2005a, 2005b).

The 3-D numerical flow model reproduced a 1:25-scale physical model studied by ENSR International (2005). The physical model is a section model of the Lower Monumental Dam having three spillway bays. The RSW was placed in the center spillway bay. The computational issue addressed in this study is whether ADH can successfully simulate the free-surface flow approaching and overtopping the RSW. This test compares a solution from the numerical model to observed laboratory data.

The ADH Navier-Stokes code employs a moving-mesh method similar to that described by Behr (2001). ADH uses the space-time finite element approach to capture the time-dependent free surface. This is essentially an ALE method of describing the fluid dynamics. The mesh nodes are restricted to move only in the vertical direction. Given a specified pressure at the free surface (typically zero), the kinematic boundary condition is satisfied and the displacement of free-surface nodes is computed. That is, the unknown at free-surface nodes is displacement rather than pressure. A displacement of the free surface is computed each time-step. To help preserve mesh quality, nodes on the interior of the domain are moved (vertically) as a function of the free-surface displacement. Certain nodes are chosen as fixed thus preserving the geometry of the dam (lower portions of the RSW, the RSW crest, spillway pier bases, etc.).

Steady state is established from an initial condition of a quiescent pool and an assumed pool elevation. Flow is first established with a fixed mesh (rigid lid) then the mesh is allowed to move and the model is run further in time until steady state with the free surface conforming to the condition of zero pressure on the water surface.

The flow boundaries, including the geometric features of the spillway piers and the RSW, were constructed in a computer-aided design (CAD) model. This CAD description of the geometry was then used as input for the grid generator. The 3-D volume mesh is of the unstructured type using tetrahedral elements. Figures 1 and 2 are scenes of the surface mesh making up the model. Three spillway bays were reproduced to simulate the physical model. The computational mesh developed to model the 1:25-scale sectional model consisted of 78,300 nodes and 412,668 tetrahedral elements. The numerical model was run at laboratory scale $(1: 25)$, but the results in this report are 
given in prototype units as they are in the ENSR report (ENSR International 2005). The surface meshes shown in Figures 1 and 2 partially illustrate the resolution of the computational mesh. The surface mesh is composed of the individual faces of the tetrahedral elements that form the boundary.

A forebay water-surface elevation of $164.6 \mathrm{~m}(540 \mathrm{ft})$ and a discharge of $265.6 \mathrm{cu} \mathrm{m} / \mathrm{sec}$ $(9,375 \mathrm{cfs})$ were simulated. The boundary conditions consist of setting flow rates at the inflow, at the outflow, at the water surface, and at all solid boundaries comprising the computational domain. Boundary conditions at the solid boundaries prohibit the flux of mass and momentum through the boundary. This no-flux boundary condition is applied to the solid boundaries and a drag coefficient is assigned to the faces of each element forming the solid boundaries. The water surface is modeled as a free surface that moves in space. This requires that the displacement be computed and that the pressure at nodes on the water surface be specified. A stress-free condition is applied for the tangential component of shear at the water surface.

The average velocity was specified uniformly across the upstream boundary. Nodes along the water surface at the inflow boundary were fixed and the pressure at these nodes was set to zero. Outflow discharge was set as natural boundary condition through each outflow face. Nodes along the water surface at the outflow were allowed to move. The pressure at each node on the water surface was specified as zero when they were allowed to move.

RESULTS: Comparisons of the ADH results and the laboratory data are provided in Figures 3-6. The observed laboratory data are labeled as ENSR data in the figures and computed results are labeled ADH. Water-surface comparisons are presented in Figure 3. The profile along the center line shows that the computed drawdown is close to that measured in the physical model. Figure 4 is a plot of the observed and computed pressure along the face (bed) of the RSW. These pressures are presented as pressure head elevations. The numerical model accurately captures the pressure variations as the flow approaches the RSW crest. This is where the flow lines are converging (Figure 7). However, after the flow passes over the crest, the streamlines appear to diverge (Figure 7), and the computed pressure magnitudes are greater than those measured. This might be attributed to the choice of eddy viscosity or the fact that the outflow boundary is too close to this area. The outflow boundary location was chosen so that flow approaching and overpassing the RSW crest could be accurately modeled. The accurate modeling of pressures on the RSW face downstream of the crest was not considered as important for this study as the accurate reproduction of the velocity approaching the weir.

The computed and the measured velocities can be compared by examining the velocity vectors plotted in Figure 5. In general, the comparisons are favorable and show the computational model does a good job of computing the velocity vectors. Observed and computed velocity magnitude contours shown in Figure 6 illustrate the velocity distribution along a vertical plane placed perpendicular to the axis of the dam at the center of the RSW. The solution is shown in the scene provided in Figure 8, which is an oblique view of the flow approaching and overtopping the RSW. The ceiling of the domain was found by the moving mesh which conforms to the shape of the free surface. The stream lines show that the flow is $3-\mathrm{D}$, having both lateral and vertical components as a particle moves in the longitudinal direction over the weir. 
CONCLUSIONS: This CHETN describes the findings of a 3-D numerical model study of flow approaching and passing over a weir. The Navier-Stokes module of the ADH code was employed with a moving-mesh algorithm to capture the position of the free surface as flow overtops the weir. Computational model results are compared to data obtained on a physical model. The comparisons show that the 3-D moving-mesh model reproduces the flow approaching and overtopping a weir with reasonable accuracy.

FUTURE WORK: The water-surface displacement in this example is both smooth and singlevalued. Nevertheless, several weaknesses in the simulator were discovered through numerical experimentation, including outright failures of the nonlinear solver algorithm under certain choices of time-step, preconditioner, and mesh parameters. Jacobian matrices in finite element computations can be ill-conditioned due simply to poor mesh quality, and this ill-conditioning leads in turn to dynamic instability. Numerical models relying on explicit parameterizations of a free surface (e.g., moving-mesh and ALE formulations) are prone to develop this type of meshinduced instability (Sethian 1999). For this reason, and because even more complex free surface and fluid-structure problems are widespread, the ADH team is developing, independently of this investigation, a set of robust and accurate tools for implicit moving boundary problems. These methods, along with hybrid methods that can maintain a high quality explicit surface mesh, will reduce the effort and increase the accuracy for simulations such as this one.

POINTS OF CONTACT: This CHETN was developed within the Navigation Systems Research and Development Program administered by James E. Clausner, Coastal and Hydraulics Laboratory, U.S. Army Engineer Research and Development Center, 3909 Halls Ferry Road, Vicksburg, MS 39180. For additional information contact Drs. Richard L. Stockstill (Richard.L.Stockstill@erdc.usace.army.mil), Christopher Kees (Christopher.E.Kees@erdc.usace. army.mil), or Charlie Berger (Charlie.R.Berger@erdc.usace.army.mil), all of the Coastal and Hydraulics Laboratory.

This CHETN should be cited as follows:

Stockstill, R. L., C. E. Kees, and R. C. Berger. 2006. Modelingfreesurface flow over a weir. ERDC/CHL CHETN-XIII-1, Vicksburg, MS: U.S. Army Engineer Research and Development Center. http://chl.erdc.usace.army.mil/chetn/.

\section{REFERENCES}

Aliabadi, S., J. Abedi, and B. Zellars. 2003. Parallel finite element simulation of mooring forces on floating objects. International Journal of Numerical Methods in Fluids. 41: 809-822.

Behr, M. 2001. Stabilized space-time finite element formulations for free-surface flows. Communications in Numerical Methods in Engineering. 17(11): 813-819.

Chang, Y. C., T. Y. Hou, B. Merriman, and S. Osher. 1996. A level set formulation of Eulerian interface capturing methods for incompressible fluid flows. Journal of Computational Physics. 124: 449-464.

Donea, J. 1982. An arbitrary Lagrangian-Eulerian finite element method for transient fluid-structure interactions. Computational Mechanics. 33: 689-723. 
ENSR International. 2005. Hydraulic model study of removable spillway weir for juvenile fish passage at Lower Monumental Dam. Report, Document No. 09000-365-2720.

Farhat, C., M. Lesoinne, and N. Maman. 1995. Mixed explicit/implicit time integration of coupled aeroelastic problems: Three-field formulation, geometric conservation and distributed solution. International Journal of Numerical Methods in Fluids. 21: 807-835.

Gulcat, U., A. Misirlioglu, and A. R. Asian. 1997. 3-D flow calculations with an A-L-E description using space-time finite elements. Communications in Numerical Methods in Engineering. 13(4): 273-284.

Hirt, W., and B. D. Nichols. 1981. Volume of fluid (VoF) method for the dynamics of free boundaries. Journal of Computational Physics. 39: 201-225.

Lin, C.-L., H. Lee, T. Lee, and L. J. Weber. 2005. A level set characteristic Galerkin finite element method for free surface flows. International Journal of Numerical Methods in Fluids. 49: 521-547.

Osher, S., and J. A. Sethian. 1988. Fronts propagating with curvature dependent speed: Algorithms based on Hamilton-Jacobi formulations. Journal of Computational Physics. 79: 12-49.

Sethian, J. A. 1999. Level set methods and fast marching methods. Cambridge monographs on applied and computational mathematics, Cambridge, MA: Cambridge University Press.

Stockstill, R. L., J. E. Hite, and J. M. Vaughan. 2005. Computational model of Ice Harbor forebay, Washington. Technical Report ERDC/CHL TR-05-5, Vicksburg, MS: U.S. Army Engineer Research and Development Center.

Stockstill, R. L., J. E. Hite, and J. M. Vaughan. 2005. Computational model of Lower Monumental forebay. Technical Report ERDC/CHL TR-05-9, Vicksburg, MS: U.S. Army Engineer Research and Development Center.

Sussman, M., P. Smareka, and S. Osher. 1994. A level set approach for computing incompressible two-phase flows. Journal of Computational Physics. 114: 146-168.

Tezduyar, T. E., M. Behr, and J. Liou. 1992. A new strategy for finite element computations involving moving boundaries and interfaces - the deforming-spatial-domain/space-time procedure: I. The concept and the preliminary tests. Computer Methods in Applied Mechanics and Engineering. 94: 339-351. 


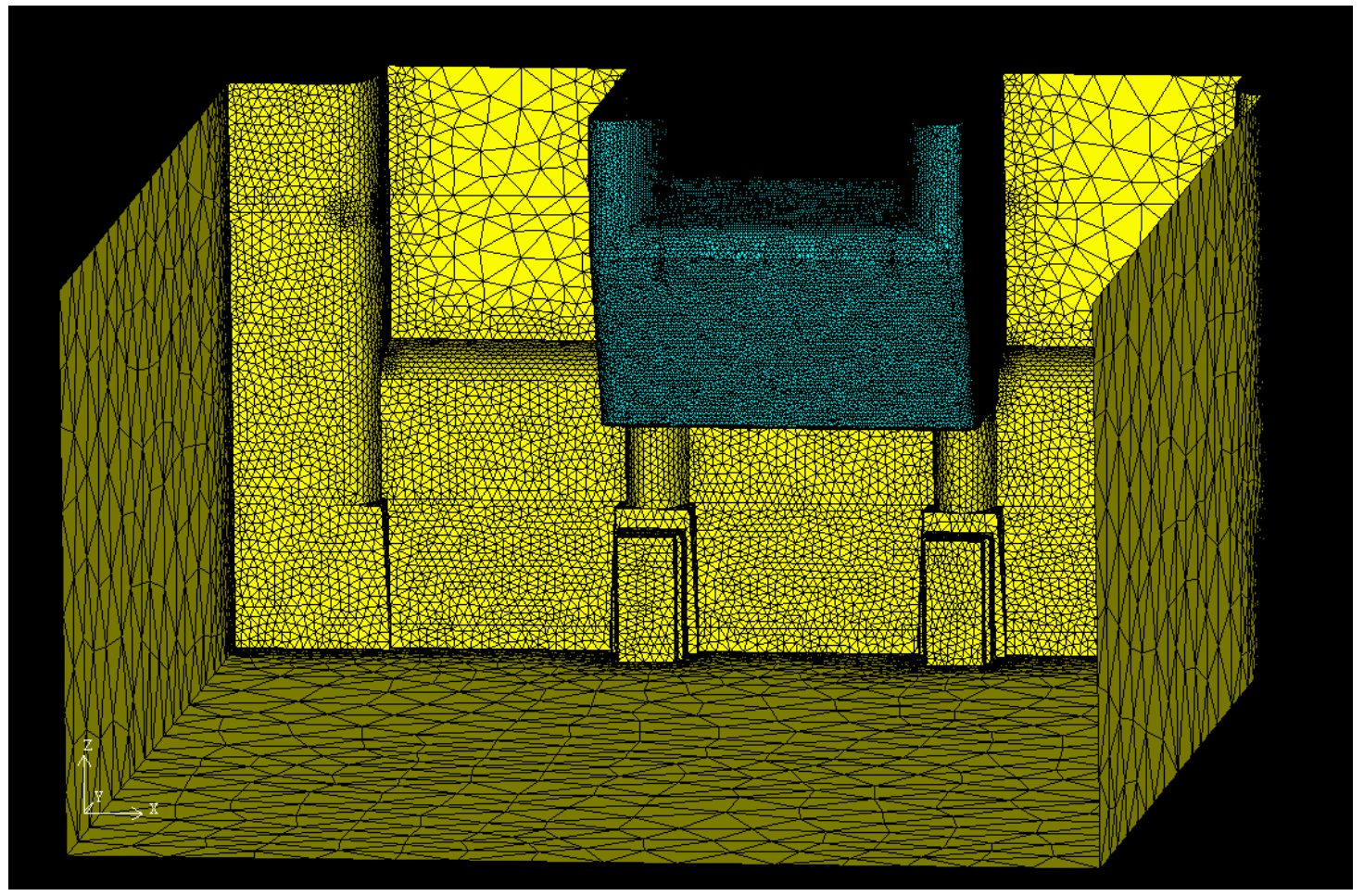

Figure 1. Overall view of surface mesh of Lower Monumental section model (inflow and water-surface boundaries removed).

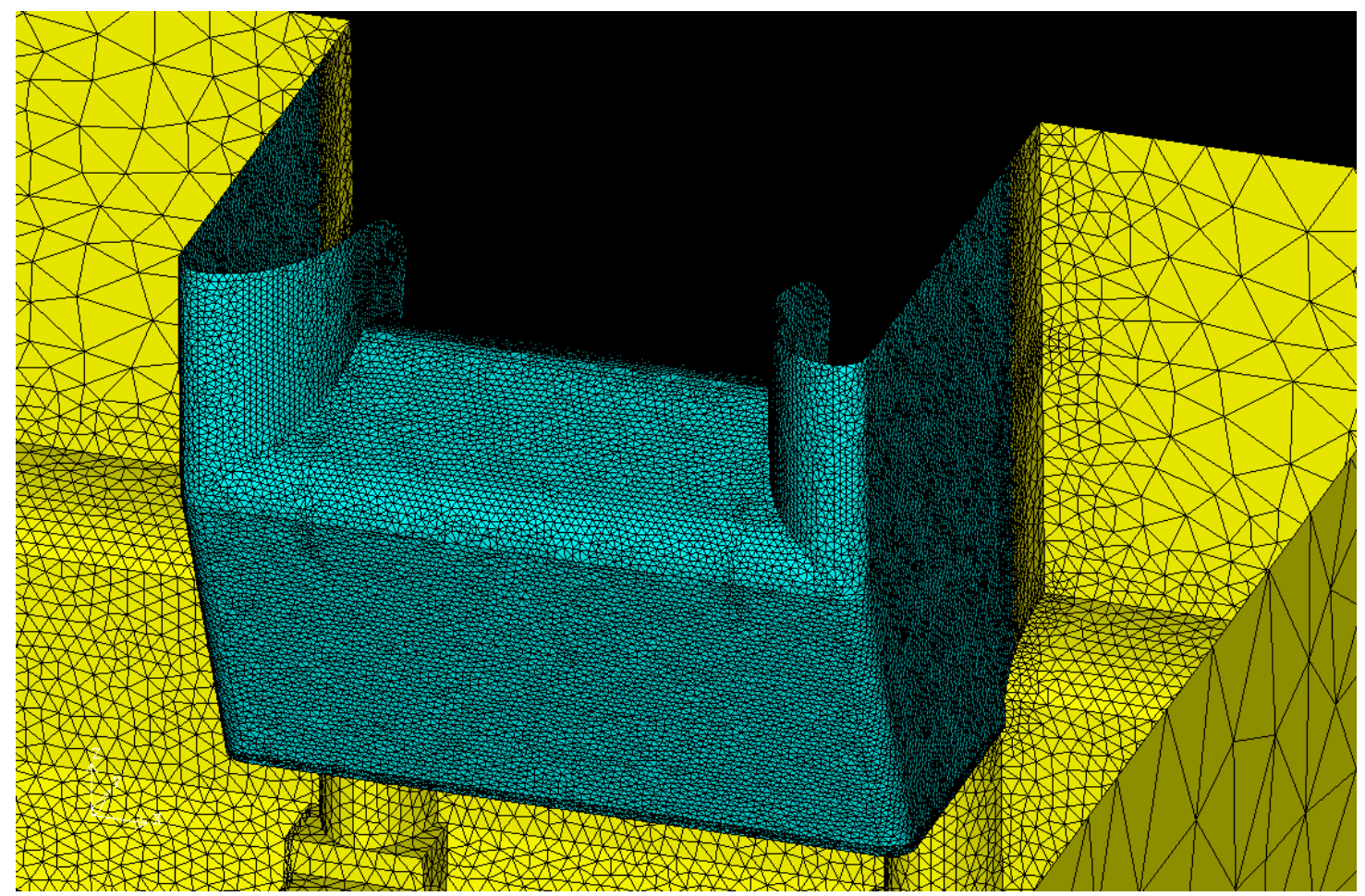

Figure 2. Close-up view of surface mesh of RSW (outflow and water-surface boundary removed). 


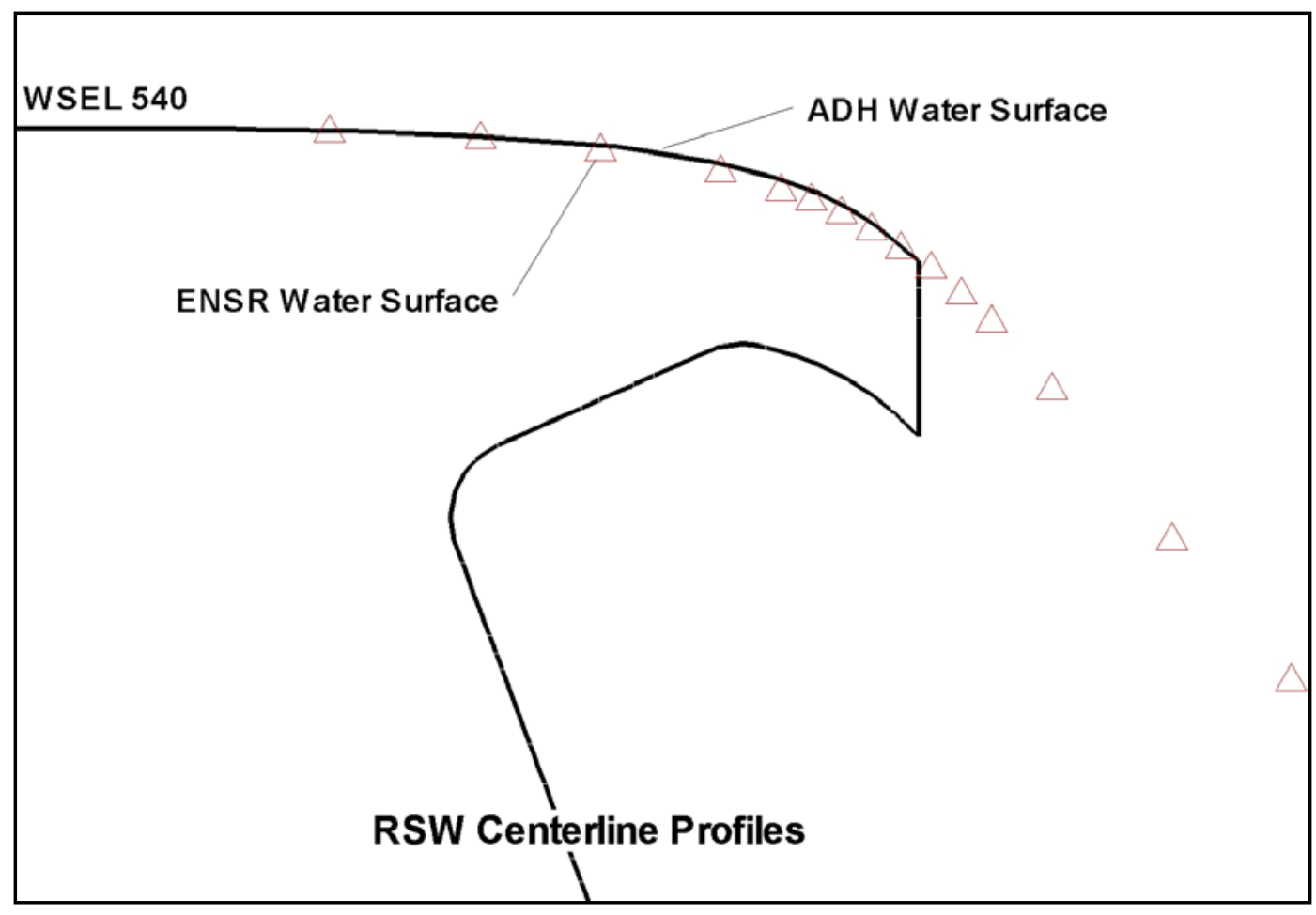

Figure 3. Comparison of computed (ADH) and observed (ENSR) water-surface profile along center line of RSW.

WSEL 540

- ADH Pressure Elevations ENSR Pressure Elevations

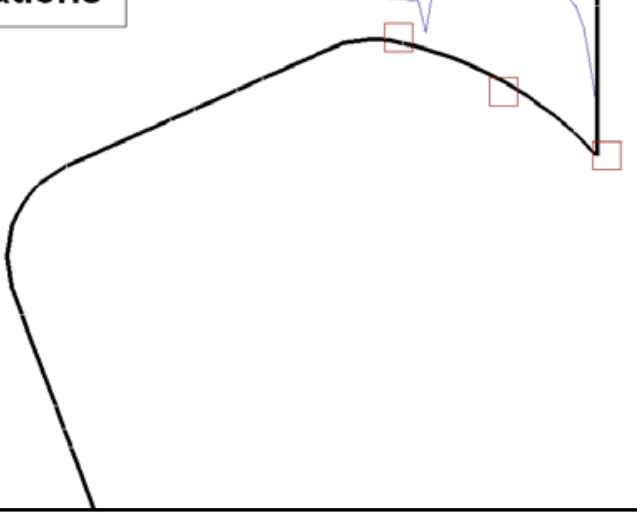

Figure 4. Comparison of computed (ADH) and observed (ENSR) pressure elevations. 


\section{WSEL 540}

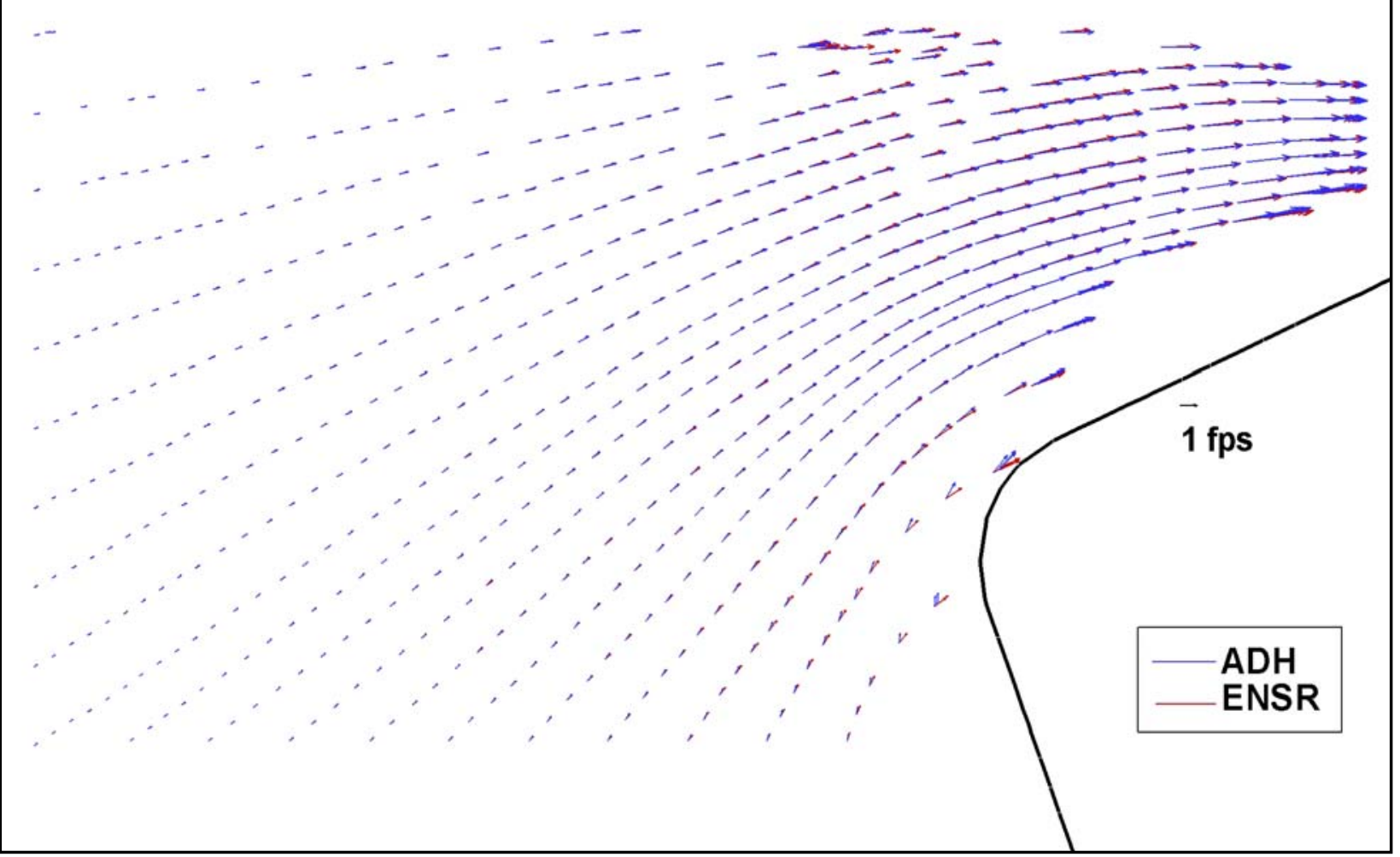

Figure 5. Comparison of computed (ADH) and observed (ENSR) velocity vectors. 


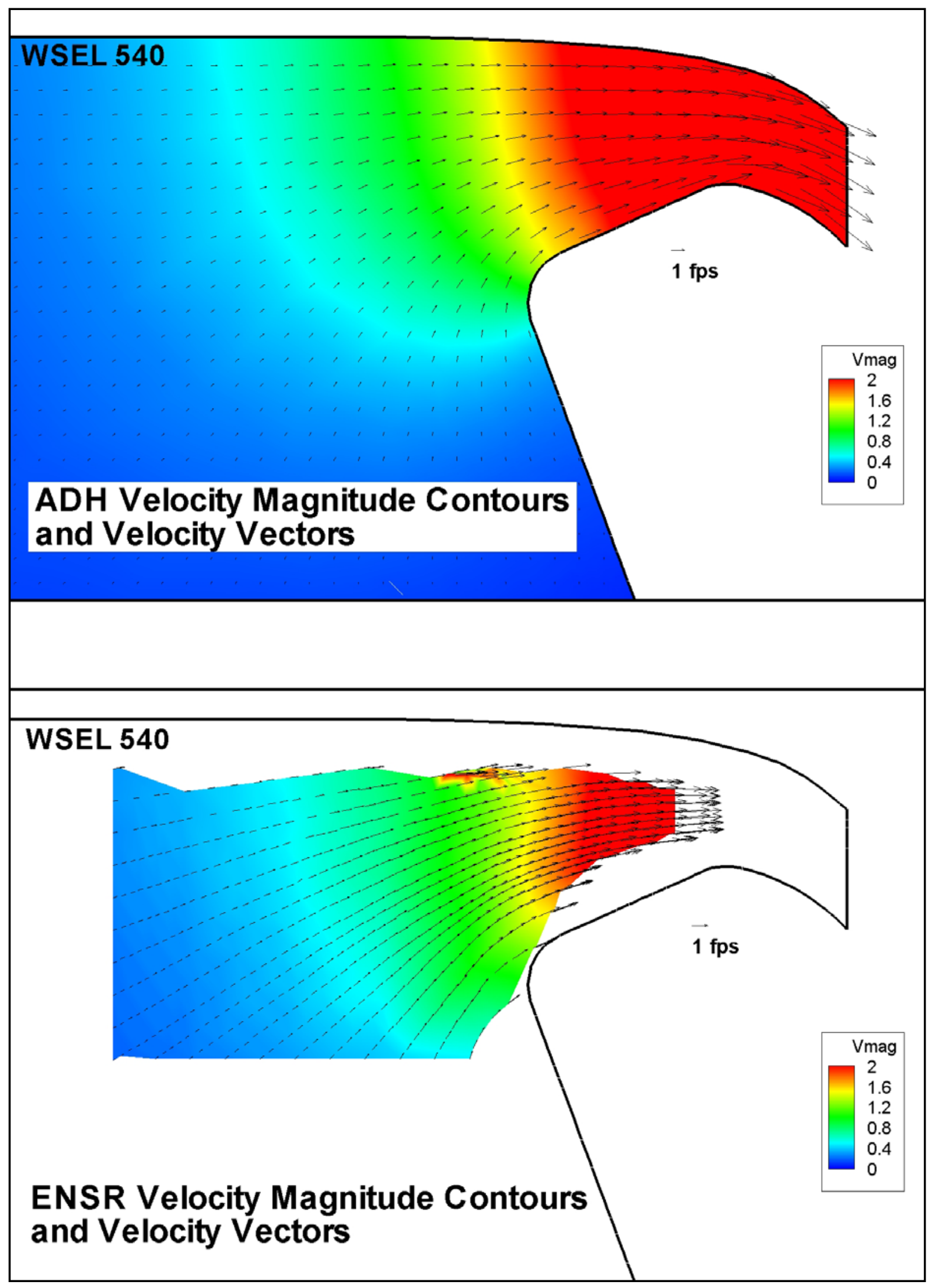

Figure 6. Comparison of computed (ADH) and observed (ENSR) velocity magnitude contours. 
ERDC/CHL CHETN-XIII-1

August 2006

\section{WSEL 540}

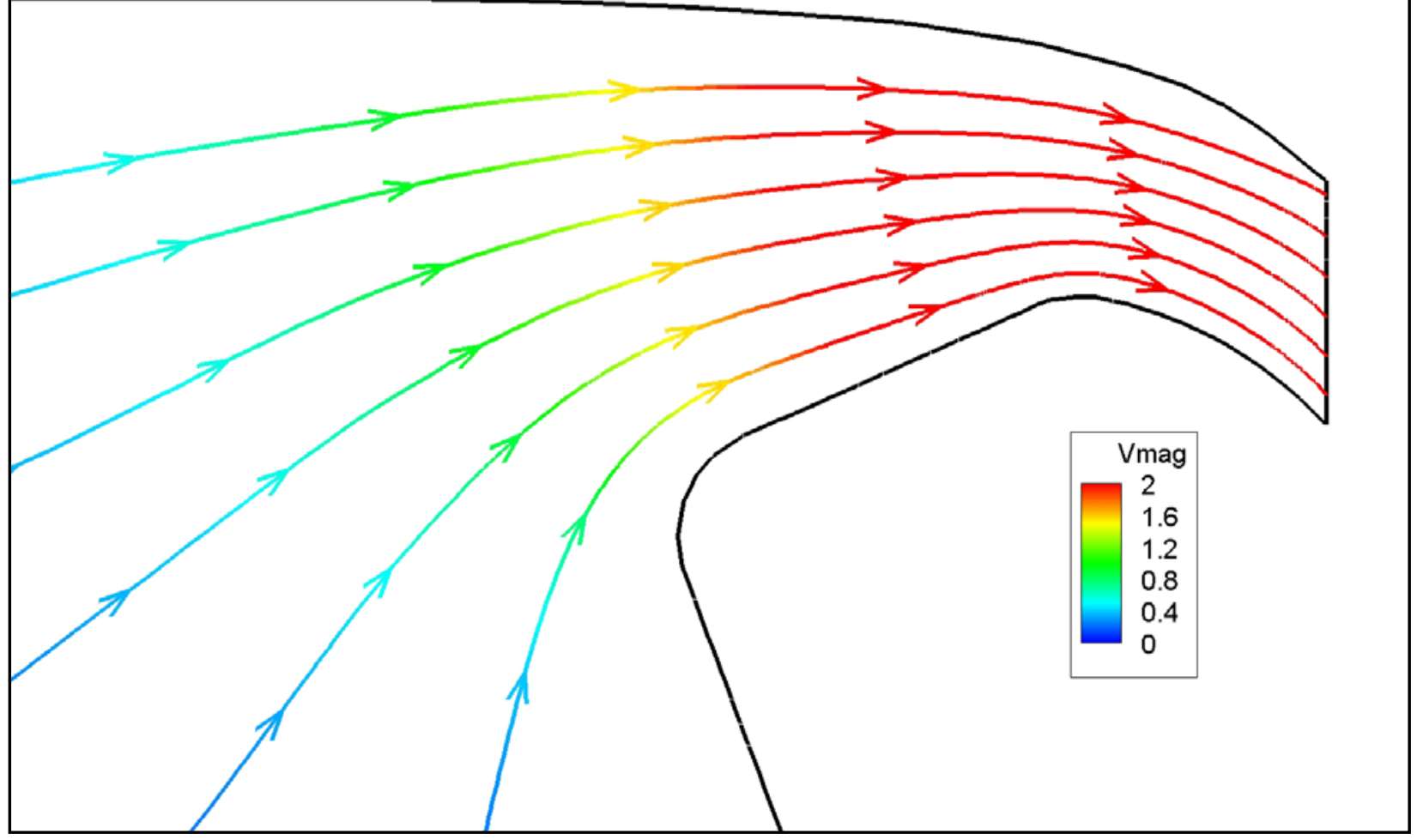

Figure 7. ADH flow over RSW. 


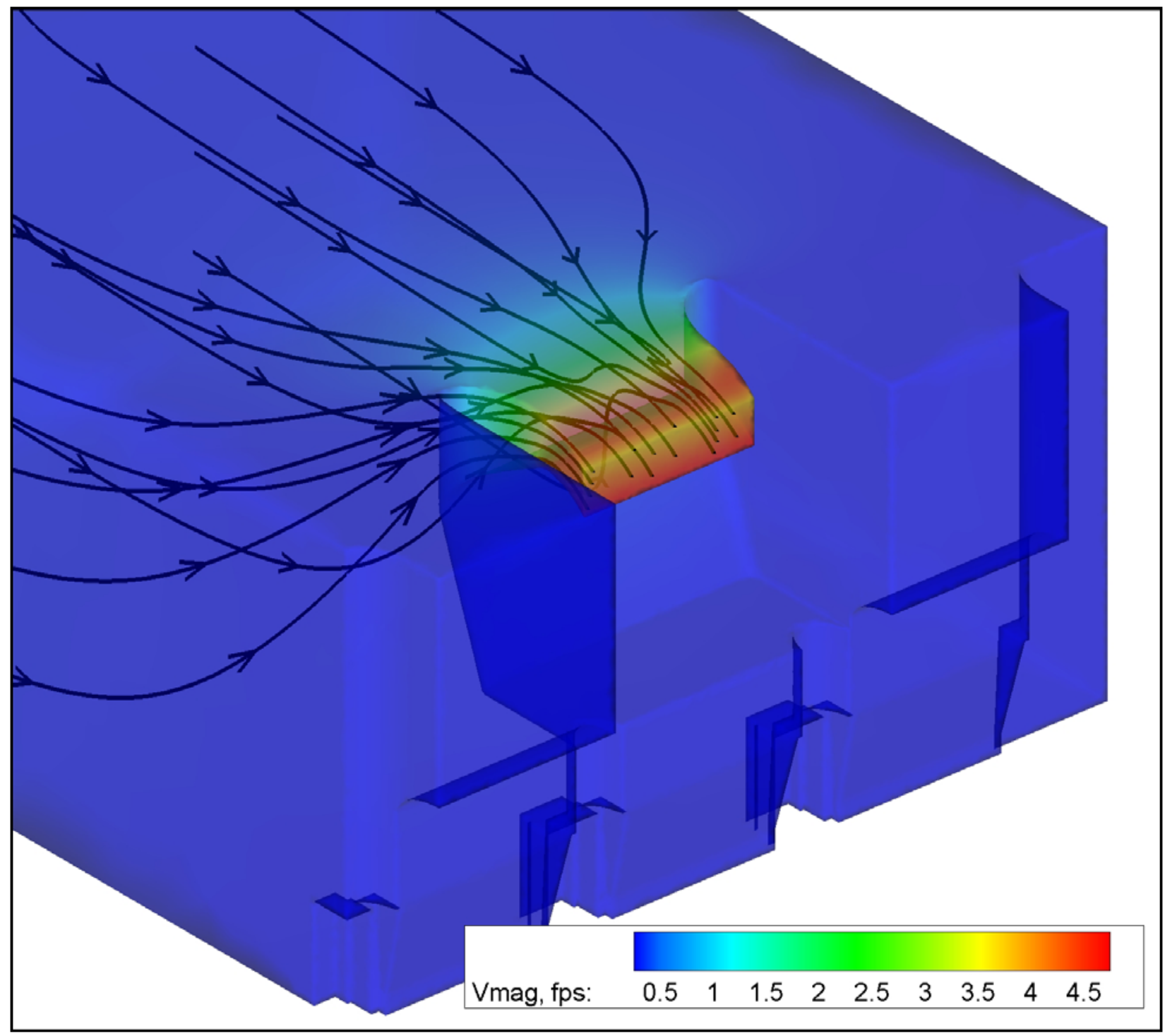

Figure 8. Velocity magnitude contours and stream traces of flow over RSW.

NOTE: The contents of this technical note are not to be used for advertising, publication, or promotional purposes. Citation of trade names does not constitute an official endorsement or approval of the use of such products. 